\title{
Small Bowel Obstruction Secondary to Acute Pancreatitis
}

\author{
Tagore Sunkara $^{\text {a, d }}$, Denzil Etienne ${ }^{\mathrm{a}}$, Megan E. Caughey ${ }^{\mathrm{b}}$, Vinaya Gaduputic
}

\begin{abstract}
While an uncommon occurrence, it is possible for patients diagnosed with acute pancreatitis to develop colonic ileus, obstruction, or perforation. By extension, it is also possible to develop a small bowel obstruction following an episode of acute pancreatitis. Here, we present the case of a 44-year-old male, who after repeated attacks of acute pancreatitis, came to the emergency department with continuous, non-bloody vomiting. This patient also complained of both left upper quadrant and epigastric pain, and was subsequently diagnosed with a small bowel obstruction involving the proximal jejunum.
\end{abstract}

Keywords: Bowel obstruction; Colon obstruction; Ileus; Pancreatitis

\section{Introduction}

In rare event acute pancreatitis leads to colonic obstructions, and it most often does so in the locations of the splenic flexure and transverse colon [1]. Small bowel obstructions following an episode of acute pancreatitis are even less documented in the literature, and tend to be the result of retroperitoneal inflammation. The small bowel is susceptible to the inflammation because of its proximity to the anterior surface of the pancreas. An enzyme-rich extravasation product, released in response to the inflammatory process, travels to the colon and small bowels, mechanically obstructing bowel pathways [2].

\section{Case Report}

A 44-year-old man with medical history of alcohol depend-

Manuscript accepted for publication January 09, 2017

aDepartment of Gastroenterology and Hepatology, The Brooklyn Hospital Center, Clinical Affiliate of The Mount Sinai Hospital, 121 Dekalb Ave., Brooklyn, NY 11201, USA

${ }^{b}$ New York Institute of Technology College of Osteopathic Medicine, Old Westbury, NY, USA

'Department of Gastroenterology and Hepatology, SBH Health System, 4422 Third Ave., Bronx, NY 10457, USA

${ }^{\mathrm{d} C}$ Corresponding Author: Tagore Sunkara, Department of Gastroenterology and Hepatology, The Brooklyn Hospital Center, Clinical Affiliate of The Mount Sinai Hospital, 121 Dekalb Avenue, Brooklyn, NY 11201, USA. Email: tagoresunkara@hotmail.com

doi: https://doi.org/10.14740/gr758w ence with recurrent attacks of acute pancreatitis came to the emergency department complaining of persistent non-bloody, greenish vomiting associated with epigastric and left upper quadrant pain for 3 days. Patient admitted to drinking alcohol daily for about 20 years. Patient had pertinent history of an episode of alcohol-induced acute pancreatitis 10 days prior to this index admission with amylase and lipase levels of 91 and 189 U/L, respectively. Patient was hemodynamically stable at the time of admission. Physical exam was consistent with epigastric and left upper quadrant tenderness without guarding or rigidity. Laboratory studies revealed a hematocrit of $43 \%$, leukocyte count of $10.3 \times 10^{9} / \mathrm{L}$ (71\% neutrophils), lipase of $163 \mathrm{U} / \mathrm{L}$, amylase of $90 \mathrm{U} / \mathrm{L}$ and creatinine of $2.7 \mathrm{mg} / \mathrm{dL}$ (baseline of $0.5 \mathrm{mg} / \mathrm{dL}$ ). Ultrasound (US) of abdomen did not reveal gallstones but showed mild hepatomegaly and hepatic steatosis. Computerized tomography (CT) scan of abdomen revealed changes in pancreas consistent with acute pancreatitis along with distended stomach and duodenum raising concern for obstruction at the proximal jejunum (Figs. 1 and 2) from surrounding edema/inflammation. Patient was admitted with an impression of alcohol-induced severe acute pancreatitis with acute kidney injury and small bowel obstruction.

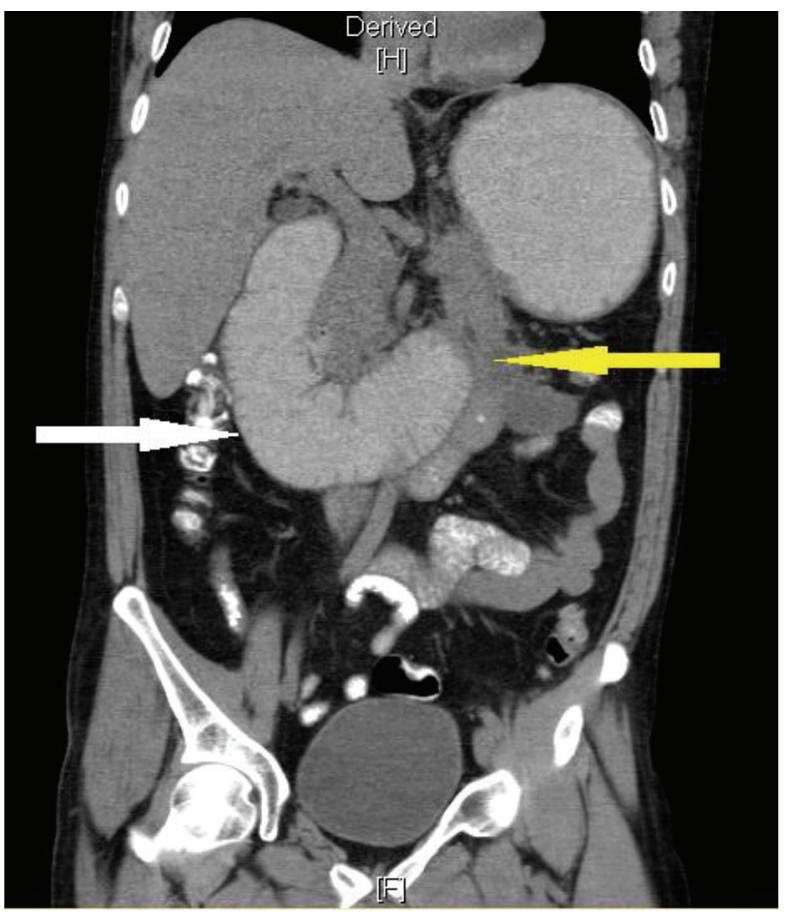

Figure 1. CT of abdomen coronal section showing severely dilated duodenum (white arrow) and peri-pancreatic fat stranding (yellow arrow). 


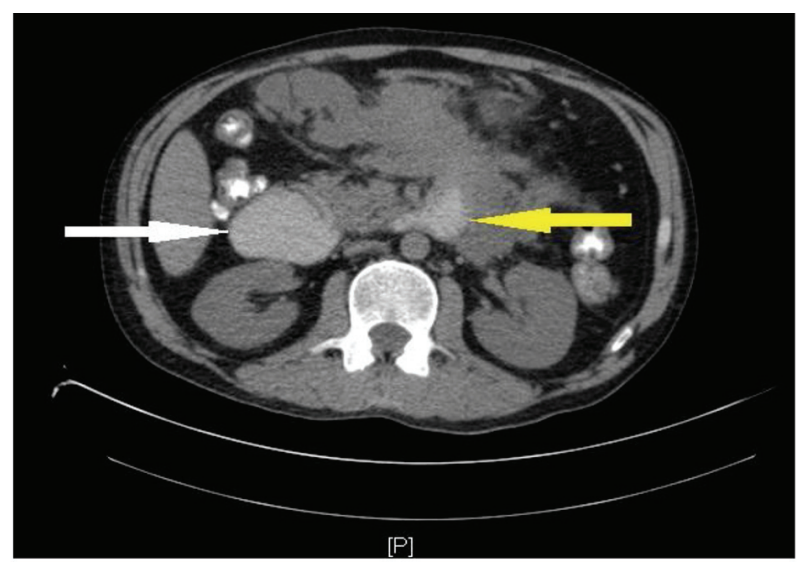

Figure 2. CT of abdomen transverse section showing severely dilated proximal duodenum (white arrow) and peri-pancreatic fat stranding (yellow arrow).

Patient was started on aggressive intravenous hydration with lactated Ringer's solution and metoclopramide as needed for nausea and vomiting. Electrolytes were replenished as appropriate. Surgeons recommended conservative management with periodic gastric suctioning via a nasogastric tube connected to low wall suction. Patient continued to have abdominal pain with nausea and vomiting over the next 2 days with worsening of leukocyte count and renal function (creatinine trended up to $4.9 \mathrm{mg} / \mathrm{dL}$ ). Patient had no bowel movements during this period. Patient was also noted to have metabolic alkalosis with a pH of 7.62. Patient was switched to intravenous normal saline given the metabolic alkalosis likely from vomiting.

Magnetic resonance cholangiopancreatography (MRCP) was also performed. MRCP revealed changes consistent with acute pancreatitis with no intra-luminal common bile duct (CBD) or pancreatic duct (PD) pathology. Patient was continued on aggressive intravenous hydration with clinical improvement and complete resolution of small bowel obstruction within next 4 days. Patient was eventually started on oral diet which he tolerated well. Patient was discharged home with extensive counseling on importance of alcohol abstinence.

\section{Discussion}

Bowel complications of acute pancreatitis such as paralytic ileus, ischemic necrosis, perforation and mechanical obstruction are relatively infrequent $[3,4]$. Mechanical bowel obstruction as a result of acute pancreatitis has been described in the literature and is more likely to occur in the splenic flexure and transverse colon. This is believed to stem from either: 1) severe inflammation of the body and tail of the pancreas causing extrinsic compression; 2) retroperitoneal extravasation of pancreatic enzymes causing pericolitis and/or pericolic fibrosis; 3 ) thrombosis of mesenteric arteries (often associated with hypercoagulability during severe inflammatory states); or 4) infarction/ischemic necrosis of watershed areas second- ary to systemic hypotension [1, 5-7]. Retroperitoneal inflammation may also lead to the involvement of other segments of bowel including the small intestine as was the case with our patient. Small bowel obstruction has not been frequently described in the literature but most likely involves similar pathogenic mechanisms described above [8-12]. Our patient represents one such case where the jejunum was involved, resulting in the clinical manifestations of small bowel mechanical obstruction.

Other obstructive manifestations of acute pancreatitis include colonic stenosis which may present as a "pseudocarcinoma" with classic "apple-core" appearance on imaging a few months following an episode of acute pancreatitis $[1,13]$. This complication has been most frequently described at the splenic flexure and, like mechanical obstruction, may be the result of the intimate anatomic relationship shared between the pancreatic tail and splenic flexure. The pancreatic tail lies in the phrenolienal and phrenocolonic ligament, which is contiguous with the splenic flexure of the colon. This shared communication facilitates direct extension of inflammatory enzyme-rich material to the splenic flexure with progressive stricturing of the colon segment [14, 15]. Additionally, the splenic flexure is a watershed area often supplied by a poorly developed marginal artery making this area more vulnerable to ischemic insult [1].

Similar anatomic relationship exists between the anterior surface of the pancreas and transverse colon where the two layers of the transverse mesocolon cover the head and body of the pancreas. Thus, enzyme-rich inflammatory extravasation can easily access the transverse colon leading to local complications (including mechanical obstruction). Furthermore, the peritoneal reflection from the anterior surface of the pancreas facilitates communication to the small bowel mesentery making the small bowel vulnerable to inflammatory complications $[2,16]$.

Finally, colonic paralytic ileus is a relatively more common and less severe complication of acute pancreatitis than true mechanical obstruction. The etiology of ileus is not entirely understood but may arise from a viscerally mediated reflex within the superior mesenteric plexus secondary to retroperitoneal inflammation and/or transient colonic ischemia [1].

Colonic obstruction secondary to acute pancreatitis usually resolves with conservative management unless it is associated with retroperitoneal collections or necrotizing pancreatitis, in which case prompt surgical intervention is warranted [17, 18]. There is paucity of literature on mechanical small bowel complications from acute pancreatitis but likely has similar pathophysiological mechanisms and management when compared to colonic complications.

\section{Conclusion}

Clinicians must be keenly aware of mechanical small bowel complications of acute pancreatitis which albeit rare, are potentially deadly and therefore should be managed aggressively. The management is directed towards treating the underlying pancreatitis and surgical management is usually reserved for 
unstable patients with refractory bowel obstruction.

\section{Funding}

This research received no specific grant from any funding agency in the public, commercial, or not-for-profit sectors.

\section{Conflicts of Interest}

The authors do not have any conflicts of interest or financial relationships to disclose.

\section{Author Contributions}

Conception and design, drafting, critical revision for important intellectual content, and final approval: Tagore Sunkara, Denzil Etienne, Megan E. Caughey, and Vinaya Gaduputi.

\section{References}

1. Aldridge MC, Francis ND, Glazer G, Dudley HA. Colonic complications of severe acute pancreatitis. Br J Surg. 1989;76(4):362-367.

2. Gardner A, Gardner G, Feller E. Severe colonic complications of pancreatic disease. J Clin Gastroenterol. 2003;37(3):258-262.

3. Russell JC, Welch JP, Clark DG. Colonic complications of acute pancreatitis and pancreatic abscess. Am J Surg. 1983;146(5):558-564.

4. Lukash WM, Bisp RP. Acute pancreatitis affecting the transverse colon. Report of a case. Am J Dig Dis. 1967;12(7):734-736.

5. Yoo SS, Choi SK, Lee DH, Jeong S, Park SH, Chung YK, Kim HG, et al. [A case of colon obstruction developed as a complication of acute pancreatitis]. Korean J Gastroenterol. 2008;51(4):255-258.
6. Miln DC, Barclay TH. Acute colonic obstruction due to pancreatitis. Lancet. 1952;2(6726):168-169.

7. Pyun DK, Kim KJ, Ye BD, Byeon JS, Myung SJ, Yang SK, $\mathrm{Kim} \mathrm{JH}$, et al. [Two cases of colonic obstruction after acute pancreatitis]. Korean J Gastroenterol. 2009;54(3):180-185.

8. Agrawal NM, Gyr N, McDowell W, Font RG. Intestinal obstruction due to acute pancreatitis. Case report and review of literature. Am J Dig Dis. 1974;19(2):179-185.

9. Brust R, Jr., Chen KC. Acute hemorrhagic pancreatitis complicated by duodenalobstruction. Report of a case. Am J Roentgenol Radium Ther Nucl Med. 1962;87:732735 .

10. Sheikh H. Duodenal Ischaemia Complicating Acute Pancreatitis. Br Med J. 1965;1(5449):1539-1540.

11. Street DF. Duodenal obstruction associated with acute pancreatitis of traumatic origin. Br J Radiol. 1959;32:617619.

12. Moore TC. Jejunal obstruction as a complication of acute hemorrhagic pancreatitis; report of a case. AMA Arch Surg. 1956;73(6):977-980.

13. Carboni M, Negro P, Tuscan0 D, de Bernardinis G, Flati D, Flati G. Secondary colonic lesions in acute pancreatitis. In: Hollender LF, ed. Controversies in Acute Pancreatitis. Berlin: Springer-Ver-lag. 1982; p. 302-315.

14. Mair WS, McMahon MJ, Goligher JC. Stenosis of the colon in acute pancreatitis. Gut. 1976;17(9):692-695.

15. Grodsinsky C, Ponka JL. The spectrum of colonic involvement in pancreatitis. Dis Colon Rectum. 1978;21(1):6670.

16. Maisonnette F, Abita T, Pichon N, Lachachi F, Cessot F, Valleix D, Durand-Fontanier S, et al. Development of colonic stenosis following severe acute pancreatitis. HPB (Oxford). 2003;5(3):183-185.

17. L'Hermine C, Pringot J, Monnier JP, Bret P, Roger J, Lemaitre L, Goncette L, et al. [Colonic complications of pancreatitis. A report on 39 cases (author's transl)]. J Radiol. 1980;61(1):27-34.

18. Gloor B, Uhl W, Muller CA, Buchler MW. The role of surgery in the management of acute pancreatitis. Can J Gastroenterol. 2000;14(Suppl D):136D-140D. 